\title{
Volume Integral Equations for Scattering from Anisotropic Diffraction Gratings
}

\author{
Armin Lechleiter* $\quad$ Dinh-Liem Nguyen ${ }^{\dagger}$
}

November 8, 2018

\begin{abstract}
We analyze electromagnetic scattering of TM polarized waves from a diffraction grating consisting of a periodic, anisotropic, and possibly negative-index dielectric material. Such scattering problems are important for the modelization of, e.g., light propagation in nano-optical components and metamaterials. The periodic scattering problem can be reformulated as a strongly singular volume integral equation, a technique that attracts continuous interest in the engineering community, but rarely received rigorous theoretic treatment. In this paper we prove new (generalized) Gårding inequalities in weighted and unweighted Sobolev spaces for the strongly singular integral equation. These inequalities also hold for materials for which the real part takes negative values inside the diffraction grating, independently of the value of the imaginary part.
\end{abstract}

\section{Introduction}

We consider scattering of time-harmonic electromagnetic waves from diffraction gratings. These three-dimensional dielectric structures are periodic in one spatial direction and invariant in a second, orthogonal, direction (compare Figure 1). They are used as optical components, e.g., to split up light into beams with different directions, and they serve in optical devices as, e.g., monochromators or as optical spectrometers.

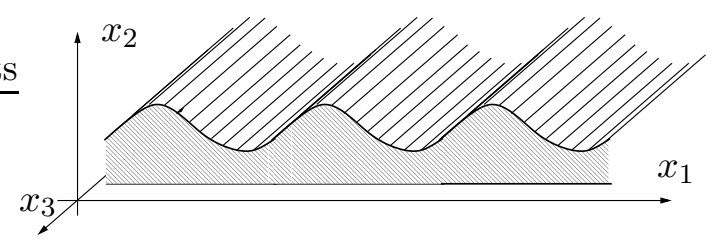

Figure 1: The diffraction grating is periodic in $x_{1}$, invariant in $x_{3}$ and bounded in $x_{2}$.

If the wave vector of an incident electromagnetic plane wave is chosen perpendicular to the invariance direction of the grating, Maxwell's equations decouple into scalar Helmholtz

${ }^{*}$ Center for Industrial Mathematics, University of Bremen, 28359 Bremen, Germany.

${ }^{\dagger}$ DEFI, INRIA Saclay-Ile-de-France and Ecole Polytechnique, 91128 Palaiseau, France. 
equations, known as transverse magnetic (TM) and transverse electric (TE) modes (these terms are not consistently used in the literature). In this paper, we consider the equation of the TM mode for a non-magnetic grating,

$$
\operatorname{div}(A \nabla u)+k^{2} u=0, \quad k>0,
$$

for an $\alpha$-quasi-periodic function $u$ (that is, $\left.u\left(x_{1}+2 \pi, x_{2}\right)=\exp (2 \pi \mathrm{i} \alpha) u\left(x_{1}, x_{2}\right)\right)$ ). In particular, we allow the real part of the discontinuous and matrix-valued material parameter $A$ to be negative-definite inside the grating structure, independently of the values of the imaginary part. (See below for the definition of the real and imaginary - or: self- and non-selfadjoint - parts of a matrix.) Negative definite material parameters are a feature that arises in the modelization of, e.g., optical metamaterials, but also for metals at certain frequencies, see, e.g., [17.

We reformulate the scattering problem using ( $\alpha$-quasi-periodic) volume integral equations. Those turn out to be strongly singular and do not fit into the standard Riesz theory, since the integral operators are not compact. Nevertheless, we prove Gårding inequalities for the integral equations in weighted $\alpha$-quasi-periodic Sobolev spaces, which yields a Fredholm framework for the scattering problem. This result even holds if the real part $\operatorname{Re}(A)$ of the material parameter is negative definite inside the grating, independently of the imaginary part $\operatorname{Im}(A)$. Our approach extends a technique from [10], where similar volume integral equations have been analyzed for free space scattering problems in case that the scalar real-valued contrast is strictly positive. Moreover, we also prove that the Gårding inequalities in weighted Sobolev spaces can be transformed to inequalities in standard $\alpha$-quasi-periodic Sobolev spaces, if the grating consists of isotropic material. Such standard Gårding inequalities are particularly useful for numerical approximation since the corresponding Galerkin methods are easier to implement in standard Sobolev spaces than in weighted spaces.

In the engineering community, volume integral equations are a popular tool to numerically solve scattering problems, see, e.g., [13,14, since they allow to solve problems with complicated material parameters via one single integral equation. However, an analysis of this technique in suitable function spaces is usually missing, in particular when the material parameters are not globally smooth, and when the arising integral operators are not compact.

Recently, volume integral equations started to attract considerable interest in applied mathematics $[5,6,8,10,12,18$. The papers [5, 10, 12, analyze strongly singular integral equations for scattering in free space. However, [12] considers media with globally continuous material properties, and the $L^{2}$-theory in [10] does not yield physical solutions if the material parameter appearing in the highest-order coefficients are not smooth. The paper [5] proves a Gårding inequality for a strongly singular volume integral equation arising from electromagnetic scattering from a (discontinuous) dielectric. Finally, 6] determines the essential spectrum of strongly singular volume integral operators arising in electromagnetic scattering for constant contrast.

The analysis of the integral equation for material parameters with negative real part is, to the best of our knowledge, the first application of $T$-coercivity (a well-known frame- 
work for variational formulations of elliptic partial differential equations with sign-changing coefficients, see [1 3]) to volume integral equations. As usual, the material parameter is, however, not allowed to take arbitrary negative values; the solvability condition for instance excludes that the relative material parameter takes the value -1 inside the grating.

The paper is organized as follows: In Section 2 we briefly recall variational theory for the direct scattering problem. In Section 3 we derive the $\alpha$-quasi-periodic volume integral equation. In Sections 5 and 4 we prove Gårding inequalities in Sobolev spaces for this equation. The two appendices contain two well-known results that do not fit comfortably into the main body of the text.

Notation: The usual $L^{2}$-based Sobolev and Lipschitz spaces on a domain $\Omega$ are denoted as $H^{s}(\Omega)$ and $C^{n, 1}(\bar{\Omega})$, respectively. Further, $H_{\mathrm{loc}}^{s}(\Omega)=\left\{v \in H^{s}(B)\right.$ for all open balls $B \subset$ $\Omega\}$. As usual, real and imaginary parts of a square matrix $A$ are defined by $\operatorname{Re} A=$ $\left(A+A^{*}\right) / 2$ and $\operatorname{Im} A=\left(A-A^{*}\right) /(2 \mathrm{i})$, where $A^{*}$ denotes the transpose conjugate matrix. Both $\operatorname{Re} A$ and $\operatorname{Im} A$ are self-adjoint and $A=\operatorname{Re}(A)+\mathrm{i} \operatorname{Im}(A)$. We denote the absolute value and the Euclidean norm by $|\cdot|$, and the spectral matrix norm by $|\cdot|_{2}$.

\section{Problem Setting}

Propagation of time-harmonic electromagnetic waves in an inhomogeneous and isotropic medium without free currents is described by the time-harmonic Maxwell's equations for the electric and magnetic fields $E$ and $H$, respectively,

$$
\operatorname{curl} H+\mathrm{i} \omega \varepsilon E=\sigma E, \quad \operatorname{curl} E-\mathrm{i} \omega \mu_{0} H=0,
$$

where $\omega>0$ denotes the angular frequency, $\varepsilon$ is the positive electric permittivity, $\mu_{0}$ is the (scalar, constant and positive) magnetic permeability, and $\sigma$ is the conductivity. The permittivity and conductivity are allowed to be anisotropic, but required to be of the special form

$$
\varepsilon=\left(\begin{array}{cc}
\varepsilon_{T} & 0 \\
0 & \varepsilon_{33}
\end{array}\right), \quad \sigma=\left(\begin{array}{cc}
\sigma_{T} & 0 \\
0 & \sigma_{33}
\end{array}\right)
$$

with real and symmetric $2 \times 2$ matrices $\varepsilon_{T}=\left(\varepsilon_{i j}\right)_{i, j=1,2}$ and $\sigma_{T}=\left(\sigma_{i j}\right)_{i, j=1,2}$, and real functions $\varepsilon_{33}$ and $\sigma_{33}$. Furthermore, we assume in this paper that all three material parameters are independent of the third variable $x_{3}$ and $2 \pi$-periodic in the first variable $x_{1}$. Moreover, $\varepsilon$ equals $\varepsilon_{0} I_{3}>0$ (where $I_{n}$ is the $n \times n$ unit matrix) and $\sigma$ equals zero outside the grating.

If an incident electromagnetic plane wave independent of the third variable $x_{3}$ illuminates the grating, then Maxwell's equations (11) for the total wave field decouple into two scalar partial differential equations (see, e.g., 7]). Indeed, since both, $E$ and $H$ do not depend on $x_{3}$ it holds that $\operatorname{curl} E=\left(\partial_{2} E_{3},-\partial_{1} E_{3}, \partial_{1} E_{2}-\partial_{2} E_{1}\right)^{\top}$ and $\operatorname{curl} H=$ $\left(\partial_{2} H_{3},-\partial_{1} H_{3}, \partial_{1} H_{2}-\partial_{2} H_{1}\right)^{\top}$. If we plug these two relations into the Maxwell's equations (11) we find that $H_{3}$ satisfies the two-dimensional scalar and anisotropic equation

$$
\operatorname{div}\left(\varepsilon_{\mathrm{r}}^{-1} \nabla u\right)+k^{2} u=0 \quad \text { in } \mathbb{R}^{2}
$$

with wave number $k:=\omega \sqrt{\varepsilon_{0} \mu_{0}}$ and material parameter

$$
\varepsilon_{\mathrm{r}}:=\varepsilon_{0}^{-1}\left[\left(\begin{array}{cc}
\varepsilon_{22} & -\varepsilon_{21} \\
-\varepsilon_{12} & \varepsilon_{11}
\end{array}\right)+\mathrm{i}\left(\begin{array}{cc}
\sigma_{22} & -\sigma_{21} \\
-\sigma_{12} & \sigma_{11}
\end{array}\right) / \omega\right] .
$$


The usual jump conditions for the Maxwell's equations imply that the field $u$ and the conormal derivative $\nu \cdot \varepsilon_{\mathrm{r}}^{-1} \nabla u$ are continuous across interfaces with normal vector $\nu$ where $\varepsilon_{\mathrm{r}}$ jumps. Note that $\varepsilon_{r}$ is $2 \pi$-periodic in $x_{1}$ and equals $I_{2}$ outside the grating.

We seek for weak solutions to (21) and assume that $\varepsilon_{\mathrm{r}} \in L^{\infty}\left(\mathbb{R}^{2}, \mathbb{C}^{2 \times 2}\right)$ takes values in the symmetric matrices, and that $\varepsilon_{\mathrm{r}}^{-1} \in L^{\infty}\left(\mathbb{R}^{2}, \mathbb{C}^{2 \times 2}\right)$. Moreover, we suppose that $\operatorname{Re}\left(\varepsilon_{\mathrm{r}}^{-1}\right)$ is pointwise strictly positive or strictly negative definite, and that $\operatorname{Im}\left(\varepsilon_{\mathrm{r}}^{-1}\right)$ is almost everywhere positive semidefinite (even if we do never exploit the latter assumption). Note that we do not assume that $\operatorname{Re}\left(\varepsilon_{\mathrm{r}}^{-1}\right)$ is positive definite in all of $\mathbb{R}^{2}$.

For the two-dimensional problem (2), incident electromagnetic waves reduce to $u^{i}(x)=$ $\exp (\mathrm{i} k x \cdot d)=\exp \left(\mathrm{i} k\left(x_{1} d_{1}+x_{2} d_{2}\right)\right)$ where $|d|=1$ and $d_{2} \neq 0$. When the incident plane wave $u^{i}$ illuminates the diffraction grating there arises a scattered field $u^{s}$ such that the total field $u=u^{i}+u^{s}$ satisfies (2). Since $\Delta u^{i}+k^{2} u^{i}=0$, the scattered field satisfies

$$
\operatorname{div}\left(\varepsilon_{\mathrm{r}}^{-1} \nabla u^{s}\right)+k^{2} u^{s}=-\operatorname{div}\left(Q \nabla u^{i}\right) \quad \text { in } \mathbb{R}^{2}, \quad \text { where } Q:=\varepsilon_{\mathrm{r}}^{-1}-I_{2}
$$

is the contrast. Note that $u^{i}$ is $\alpha$-quasi-periodic with respect $x_{1}$, that is,

$$
u^{i}\left(x_{1}+2 \pi, x_{2}\right)=e^{2 \pi \mathrm{i} \alpha} u^{i}\left(x_{1}, x_{2}\right) \quad \text { for } \alpha:=k d_{1} .
$$

Since $u^{i}$ is $\alpha$-quasi-periodic and $\varepsilon_{\mathrm{r}}$ is periodic, the total field and the scattered field both are also $\alpha$-quasi-periodic in $x_{1}$. For uniqueness of solution, the scattered field additionally has to satisfy a radiation condition. Here we require that $u^{s}$ above (below) the dielectric structure can be represented by a uniformly converging Fourier(-Rayleigh) series consisting of upwards (downwards) propagating or evanescent plane waves, see [4,9],

$$
u^{s}(x)=\sum_{j \in \mathbb{Z}} \hat{u}_{j}^{ \pm} e^{\mathrm{i} \alpha_{j} x_{1} \pm \mathrm{i} \beta_{j}\left(x_{2}-\rho\right)}, \quad x_{2} \gtrless \pm \rho, \quad \alpha_{j}:=j+\alpha, \quad \beta_{j}:=\left(k^{2}-\alpha_{j}^{2}\right)^{1 / 2},
$$

where $\rho>\sup \left\{\left|x_{2}\right|:\left(x_{1}, x_{2}\right)^{\top} \in \operatorname{supp}(Q)\right\}$. Let us set $\Gamma_{ \pm \rho}=(-\pi, \pi) \times\{ \pm \rho\}$. The numbers $\hat{u}_{j}^{ \pm}$are the so-called Rayleigh coefficients of $u^{s}$, defined by

$$
\hat{u}_{j}^{ \pm}=\frac{1}{2 \pi} \int_{\Gamma_{ \pm \rho}} u^{s}\left(x_{1}, x_{2}\right) \exp \left(-\mathrm{i} \alpha_{j} x_{1}\right) \mathrm{d} s, \quad j \in \mathbb{Z} .
$$

A solution to the Helmholtz equation is called radiating if it satisfies (4). If $k^{2}>\alpha_{j}^{2}$ then the $j$ th mode $\exp \left(i \alpha_{j} x_{1} \pm i \beta_{j}\left(x_{2} \pm \rho\right)\right)$ is a propagating mode, whereas $k^{2}<\alpha_{j}^{2}$ means that $\exp \left(i \alpha_{j} x_{1} \pm i \beta_{j}\left(x_{2} \pm \rho\right)\right)$ is an evanescent mode.

Variational solution theory for the scattering problem (3)-(4) is well-known, see, e.g., 4, 7,9. Setting

$$
\Omega_{\rho}:=(-\pi, \pi) \times(-\rho, \rho)
$$

for $\rho>\sup \left\{\left|x_{2}\right|:\left(x_{1}, x_{2}\right)^{\top} \in \operatorname{supp}(Q)\right\}$, one can variationally reformulate the problem in the space $H_{\alpha}^{1}\left(\Omega_{\rho}\right):=\left\{u \in H^{1}\left(\Omega_{\rho}\right): u=\left.U\right|_{\Omega_{\rho}}\right.$ for some $\alpha$-quasi-periodic $\left.U \in H_{\text {loc }}^{1}\left(\mathbb{R}^{2}\right)\right\}$. The resulting variational formulation is to find $u^{s} \in H_{\alpha}^{1}\left(\Omega_{\rho}\right)$ such that

$$
\begin{aligned}
\int_{\Omega_{\rho}}\left(\varepsilon_{\mathrm{r}}^{-1} \nabla u^{s} \cdot \nabla \bar{v}-k^{2} u^{s} \bar{v}\right) \mathrm{d} x-\int_{\Gamma_{\rho}} \bar{v} T^{+}\left(u^{s}\right) \mathrm{d} s & -\int_{\Gamma_{-\rho}} \bar{v} T^{-}\left(u^{s}\right) \mathrm{d} s \\
& =-\int_{\Omega_{\rho}} Q \nabla u^{i} \cdot \nabla \bar{v} \mathrm{~d} x
\end{aligned}
$$


for all $v \in H_{\alpha}^{1}\left(\Omega_{\rho}\right)$. The operators $T^{ \pm}, \varphi \mapsto \mathrm{i} \sum_{j \in \mathbb{Z}} \beta_{j} \hat{\varphi}_{j}^{ \pm} e^{\mathrm{i} \alpha_{j} x_{1}}$, are the so-called exterior Dirichlet-to-Neumann operators on $\Gamma_{ \pm \rho}$. The sesquilinear form in (5) is bounded on $H_{\alpha}^{1}\left(\Omega_{\rho}\right)$ and satisfies a Gårding inequality if, e.g., $\operatorname{Re}\left(\varepsilon_{\mathrm{r}}^{-1}\right)$ is positive definite, that is, $\xi^{*} \operatorname{Re}\left(\varepsilon_{\mathrm{r}}^{-1}\right)(x) \xi \geq c|\xi|^{2} \geq 0$ for $\xi \in \mathbb{C}^{2}$ and almost every $x \in \Omega_{\rho}$. In this case, analytic Fredholm theory implies that the set of real wave numbers (excluding Rayleigh frequencies) where non-uniqueness occurs is at most countable, see [4,9]. If $\operatorname{Re}\left(\varepsilon_{\mathrm{r}}^{-1}\right)$ changes sign, proving Fredholm properties of the variational formulation (5) is non-trivial, at least if $\operatorname{Im} \varepsilon_{\mathrm{r}}^{-1}$ vanishes.

In this paper, we establish a Fredholm framework for the scattering problem via integral equation techniques, that is, uniqueness of solution implies existence. We do not aim to prove the corresponding uniqueness results, since for periodic scattering problems such results are anyway not available at all frequencies, except under restrictive geometric (nontrapping) conditions, see [4].

\section{Integral Equation Formulation}

In this section, we reformulate the scattering problem (2) as a volume integral equation, and prove mapping properties of the integral operator between Sobolev spaces. To this end, let us recall that $Q=\varepsilon_{\mathrm{r}}^{-1}-I_{2}$ and denote by $\bar{D} \subset \Omega_{\rho}$ the support of $Q$, restricted to one period $\left\{-\pi<x_{1}<\pi\right\}$. By $G_{k, \alpha}$ we denote the Green's function to the $\alpha$-quasi-periodic Helmholtz equation in $\mathbb{R}^{2}$, see [9]. Under the assumption that

$$
k^{2} \neq \alpha_{j}^{2} \quad \text { for all } j \in \mathbb{Z},
$$

the $\alpha$-quasi-periodic Green's function has the series representation

$$
G_{k, \alpha}(x):=\frac{\mathrm{i}}{4 \pi} \sum_{j \in \mathbb{Z}} \frac{1}{\beta_{j}} \exp \left(\mathrm{i} \alpha_{j} x_{1}+\mathrm{i} \beta_{j}\left|x_{2}\right|\right), \quad x=\left(\begin{array}{c}
x_{1} \\
x_{2}
\end{array}\right) \in \mathbb{R}^{2}, x \neq\left(\begin{array}{c}
2 \pi m \\
0
\end{array}\right) \text { for } m \in \mathbb{Z} .
$$

Due to (6) all the $\beta_{j}=\left(k^{2}-\alpha_{j}^{2}\right)^{1 / 2}$ are non-zero.

Lemma 1 (Cf. [9]). The Green's function $G_{k, \alpha}$ can be split into $G_{k, \alpha}(x)=(\mathrm{i} / 4) H_{0}^{(1)}(k|x|)+$ $\Psi(x)$ in $\mathbb{R}^{2}$ where $\Psi$ is an analytic function solving the Helmholtz equation $\Delta \Psi+k^{2} \Psi=0$ in $(-2 \pi, 2 \pi) \times \mathbb{R}$.

We also define a periodized Green's function, firstly setting

$$
\mathcal{K}_{\rho}(x):=G_{k, \alpha}(x), \quad x=\left(\begin{array}{l}
x_{1} \\
x_{2}
\end{array}\right) \in \mathbb{R} \times(-\rho, \rho), x \neq\left(\begin{array}{c}
2 \pi m \\
0
\end{array}\right) \text { for } m \in \mathbb{Z},
$$

and secondly extending $\mathcal{K}_{\rho}(x) 2 \rho$-periodically in $x_{2}$ to $\mathbb{R}^{2}$.

The trigonometric polynomials

$$
\varphi_{j}(x):=\frac{1}{\sqrt{4 \pi \rho}} \exp \left(\mathrm{i}\left(j_{1}+\alpha\right) x_{1}+\mathrm{i} \frac{j_{2} \pi}{\rho} x_{2}\right), \quad j=\left(\begin{array}{c}
j_{1} \\
j_{2}
\end{array}\right) \in \mathbb{Z}^{2},
$$


are orthonormal in $L^{2}\left(\Omega_{\rho}\right)$. They differ from the usual Fourier basis (see, e.g., [16, Section 10.5.2]) only by a phase factor $\exp \left(\mathrm{i} \alpha x_{1}\right)$, and hence also form a basis of $L^{2}\left(\Omega_{\rho}\right)$. For $f \in L^{2}\left(\Omega_{\rho}\right)$ and $j=\left(j_{1}, j_{2}\right)^{\top} \in \mathbb{Z}^{2}, \hat{f}(j):=\int_{\Omega_{\rho}} f \overline{\varphi_{j}} \mathrm{~d} x$ are the Fourier coefficients of $f$. For $0 \leq s<\infty$ we define a fractional Sobolev space $H_{\text {per }}^{s}\left(\Omega_{\rho}\right)$ as the subspace of functions in $L^{2}\left(\Omega_{\rho}\right)$ such that

$$
\|f\|_{H_{\mathrm{per}}^{s}\left(\Omega_{\rho}\right)}^{2}=\sum_{j \in \mathbb{Z}^{2}}\left(1+|j|^{2}\right)^{s}|\hat{f}(j)|^{2}<\infty .
$$

It is well-known that for integer values of $s$, these spaces correspond to spaces of $\alpha$-quasiperiodic functions that are $s$ times weakly differentiable, and that the above norm is then equivalent to the usual integral norms.

Lemma 1 implies in particular that $\mathcal{K}_{\rho}$ has an integrable singularity and that the Fourier coefficients $\hat{\mathcal{K}}_{\rho}(j)$ are well-defined. To compute these coefficients explicitly, we set

$$
\lambda_{j}:=k^{2}-\left(j_{1}+\alpha\right)^{2}-\left(\frac{j_{2} \pi}{\rho}\right)^{2} \text { for } j \in \mathbb{Z}^{2} .
$$

Theorem 2. Assume that $k^{2} \neq \alpha_{j}^{2}$ for all $j \in \mathbb{Z}$. Then the Fourier coefficients of the kernel $\mathcal{K}_{\rho}$ from (8) are given by

$$
\hat{\mathcal{K}}_{\rho}(j)= \begin{cases}\frac{\cos \left(j_{2} \pi\right) e^{\mathrm{i} \beta j_{1} \rho}-1}{\sqrt{4 \pi \rho} \lambda_{j}} & \text { for } \lambda_{j} \neq 0, \quad j=\left(\begin{array}{l}
j_{1} \\
j_{2}
\end{array}\right) \in \mathbb{Z}^{2} . \\
\frac{\mathrm{i}}{4 j_{2}}\left(\frac{\rho}{\pi}\right)^{3 / 2} & \text { else, }\end{cases}
$$

Remark 3. Note that $\hat{\mathcal{K}}_{\rho}(j)$ is well-defined for $\lambda_{j}=0$ : Since $k^{2} \neq \alpha_{n}^{2}$ for all $n \in \mathbb{Z}$, the definition of $\lambda_{j}$ implies that $j_{2} \neq 0$ whenever $\lambda_{j}=0$. For completeness, we include a proof, noting that the case $\lambda_{j} \neq 0$ is also shown in [15, Section 7.1].

Proof. It is easy to check that $\left(\Delta+k^{2}\right) \varphi_{j}=\lambda_{j} \varphi_{j}$ for $j=\left(j_{1}, j_{2}\right)^{\top} \in \mathbb{Z}^{2}$. If $\lambda_{j} \neq 0$, Green's second identity implies that

$$
\begin{aligned}
& \hat{\mathcal{K}}_{\rho}(j)= \int_{\Omega_{\rho}} \mathcal{K}_{\rho}(x) \overline{\varphi_{j}(x)} \mathrm{d} x=\lambda_{j}^{-1} \lim _{\delta \rightarrow 0} \int_{\Omega_{\rho} \backslash B(0, \delta)} G_{k, \alpha}(x) \overline{\left(\Delta+k^{2}\right) \varphi_{j}(x)} \mathrm{d} x \\
&=\lambda_{j}^{-1} \lim _{\delta \rightarrow 0}\left[\left(\int_{\partial \Omega_{\rho}}+\int_{\partial B(0, \delta)}\right)\left(G_{k, \alpha} \frac{\partial \overline{\varphi_{j}}}{\partial \nu}-\frac{\partial G_{k, \alpha}}{\partial \nu} \overline{\varphi_{j}}\right) \mathrm{d} s\right. \\
&\left.+\int_{\Omega_{\rho} \backslash B(0, \delta)}\left(\Delta+k^{2}\right) G_{k, \alpha}(x) \overline{\varphi_{j}(x)} \mathrm{d} x\right],
\end{aligned}
$$

where $\nu$ denotes the exterior normal vector to $B(0, \delta)$. The last volume integral vanishes since $\left(\Delta+k^{2}\right) G_{k, \alpha}=0$ in $\Omega_{\rho} \backslash B(0, \delta)$ for any $\delta>0$. Let us now consider the first integral in (11). The boundary of $\Omega_{\rho}$ consists of two horizontal lines $\Gamma_{ \pm \rho}$ and two vertical lines $\left\{\left(x_{1}, x_{2}\right): x_{1}= \pm \pi,-\rho<x_{2}<\rho\right\}$. Hence, the normal vector $\nu$ on these boundaries is 
either $( \pm 1,0)^{\top}$ or $(0, \pm 1)^{\top}$. Straightforward computations yield that

$$
\begin{array}{cc}
G_{k, \alpha}\left(x_{1}, \pm \rho\right)=\frac{\mathrm{i}}{4 \pi} \sum_{n \in \mathbb{Z}} \frac{e^{\mathrm{i} \beta_{n} \rho}}{\beta_{n}} e^{\mathrm{i} \alpha_{n} x_{1}}, & \partial_{2} G_{k, \alpha}\left(x_{1}, \pm \rho\right)=\mp \frac{1}{4 \pi} \sum_{n \in \mathbb{Z}} e^{\mathrm{i} \beta_{n} \rho} e^{\mathrm{i} \alpha_{n} x_{1}}, \\
\overline{\varphi_{j}\left(x_{1}, \pm \rho\right)}=\frac{1}{\sqrt{4 \pi \rho}} e^{-\mathrm{i} \alpha_{j_{1}} x_{1}} \cos \left(j_{2} \pi\right), & \text { and } \quad \partial_{2} \overline{\varphi_{j}\left(x_{1}, \pm \rho\right)}=-\frac{\mathrm{i} j_{2} \pi}{\rho} \overline{\varphi\left(x_{1}, \pm \rho\right)} .
\end{array}
$$

In consequence,

$$
\begin{aligned}
\int_{\Gamma_{ \pm \rho}}\left(G_{k, \alpha} \frac{\partial \overline{\varphi_{j}}}{\partial \nu}-\frac{\partial G_{k, \alpha}}{\partial \nu} \overline{\varphi_{j}}\right) \mathrm{d} s & =-\int_{\Gamma_{\rho}} \partial_{2} G_{k, \alpha} \overline{\varphi_{j}} \mathrm{~d} s+\int_{\Gamma_{-\rho}} \partial_{2} G_{k, \alpha} \overline{\varphi_{j}} \mathrm{~d} s \\
& =-2 \int_{\Gamma_{\rho}} \partial_{2} G_{k, \alpha} \overline{\varphi_{j}} \mathrm{~d} s .
\end{aligned}
$$

Using the above formulas for $\partial_{2} G_{k, \alpha}$ and $\overline{\varphi_{j}}$ in (13) and (14), respectively, we find that

$$
-2 \int_{\Gamma_{\rho}} \partial_{2} G_{k, \alpha} \overline{\varphi_{j}} \mathrm{~d} s=\frac{\cos \left(j_{2} \pi\right)}{\sqrt{4 \pi \rho}} e^{\mathrm{i} \beta_{j_{1}} \rho} .
$$

Computing the partial derivatives of $G_{k, \alpha}$ and $\varphi_{j}$ with respect to $x_{1}$ analogously to the above computations, one finds that the integrals on the vertical boundaries of $\Omega_{\rho}$ vanish due to the $\alpha$-quasi-periodicity of both functions. Thus, we obtain that

$$
\int_{\partial \Omega_{\rho}}\left(G_{k, \alpha} \frac{\partial \overline{\varphi_{j}}}{\partial \nu}-\frac{\partial G_{k, \alpha}}{\partial \nu} \overline{\varphi_{j}}\right) \mathrm{d} s=\frac{\cos \left(j_{2} \pi\right)}{\sqrt{4 \pi \rho}} e^{\mathrm{i} \beta_{j_{1}} \rho} .
$$

Now we consider the second integral in (11). From Lemma 1 we know that $G_{k, \alpha}(x)=$ $\frac{\mathrm{i}}{4} H_{0}^{(1)}(k|x|)+\Psi(x)$ where $\Psi$ is a smooth function in $\Omega_{\rho}$. Obviously,

$$
\lim _{\delta \rightarrow 0} \int_{\partial B(0, \delta)}\left(\Psi \frac{\partial \overline{\varphi_{j}}}{\partial \nu}-\frac{\partial \Psi}{\partial \nu} \overline{\varphi_{j}}\right) \mathrm{d} s=0
$$

The asymptotics of $H_{0}^{(1)}$ and its derivative for small arguments,

$$
H_{0}^{(1)}(r)=\frac{2 \mathrm{i}}{\pi} \log r+\mathcal{O}(1) \quad \text { and } \quad\left(H_{0}^{(1)}\right)^{\prime}(r)=\frac{2 \mathrm{i}}{\pi r}+\mathcal{O}(1) \quad \text { as } r \rightarrow 0,
$$

allow to show that

$$
\lim _{\delta \rightarrow 0} \int_{\partial B(0, \delta)}\left(G_{k, \alpha} \frac{\partial \overline{\varphi_{j}}}{\partial \nu}-\frac{\partial G_{k, \alpha}}{\partial r} \overline{\varphi_{j}}\right) \mathrm{d} s,=-\frac{1}{\sqrt{4 \pi \rho}},
$$

see, e.g., [16, Theorem 2.2.1]. Combining (15) with (16) yields that

$$
\mathcal{K}_{\rho}(j)=\frac{1}{\sqrt{4 \pi \rho} \lambda_{j}}\left(\cos \left(j_{2} \pi\right) e^{\mathrm{i} \beta_{j_{1}} \rho}-1\right) \quad \text { for } \lambda_{j} \neq 0
$$


For $\lambda_{j}=0$ we use de L'Hôspital's rule to find that

$$
\mathcal{K}_{\rho}(j)=\lim _{\gamma \rightarrow\left(j_{1}+\alpha\right)^{2}+\left(j_{2} \pi / \rho\right)^{2}} \frac{\cos \left(j_{2} \pi\right) \exp \left(\mathrm{i} \rho \sqrt{\gamma-\left(j_{1}+\alpha\right)^{2}}\right)-1}{\sqrt{4 \pi \rho}\left[\gamma-\left(j_{1}+\alpha\right)^{2}-\left(j_{2} \pi / \rho\right)^{2}\right]}=\frac{\mathrm{i} \rho^{3 / 2}}{4 \pi^{3 / 2} j_{2}} .
$$

Note that the assumption that $k^{2} \neq \alpha_{j}^{2}$ for all $j \in \mathbb{Z}^{2}$ implies that $\lambda_{j}$ and $j_{2}$ cannot vanish simultaneously.

Since the Fourier coefficients of the $\mathcal{K}_{\rho}$ decay quadratically, $\left|\hat{\mathcal{K}}_{\rho}(j)\right| \leq C /\left(1+\left(j_{1}+\alpha\right)^{2}+\right.$ $\left.\left(j_{2} \pi / \rho\right)^{2}\right)$ for $j \in \mathbb{Z}^{2}$, the convolution operator with kernel $\mathcal{K}_{\rho}$ is bounded from $L^{2}\left(\Omega_{\rho}\right)$ into $H_{\text {per }}^{2}\left(\Omega_{\rho}\right)$.

Proposition 4. Assume that $k^{2} \neq \alpha_{j}^{2}$ for all $j \in \mathbb{Z}$. Then the convolution operator $K_{\rho}$, defined by

$$
\left(K_{\rho} f\right)(x)=\int_{\Omega_{\rho}} \mathcal{K}_{\rho}(x-y) f(y) \mathrm{d} y \quad \text { for } x \in \Omega_{\rho},
$$

is bounded from $L^{2}\left(\Omega_{\rho}\right)$ into $H_{\mathrm{per}}^{2}\left(\Omega_{\rho}\right)$.

Recall that $D \subset \Omega_{\rho}$ is the support of contrast $Q$. Let us additionally introduce

$$
\Omega:=(-\pi, \pi) \times \mathbb{R}
$$

and $H_{\alpha}^{\ell}\left(\Omega_{R}\right):=\left\{u \in H^{\ell}\left(\Omega_{R}\right): u=\left.U\right|_{\Omega_{R}}\right.$ for some $\alpha$-quasi-periodic $\left.U \in H_{\text {loc }}^{\ell}\left(\mathbb{R}^{2}\right)\right\}$ for $\ell \in \mathbb{N}, R>0$.

Lemma 5. Assume that $k^{2} \neq \alpha_{j}^{2}$ for all $j \in \mathbb{Z}$. Then the volume potential $V_{k}$ defined by

$$
\left(V_{k} f\right)(x)=\int_{D} G_{k, \alpha}(x-y) f(y) \mathrm{d} y, \quad x \in \Omega,
$$

is bounded from $L^{2}(D)$ into $H_{\alpha}^{2}\left(\Omega_{R}\right)$ for all $R>0$.

Proof. Consider $\chi \in C^{\infty}(\Omega)$ such that $\chi=1$ in $D, 0 \leq \chi \leq 1$ in $\Omega_{\rho} \backslash \bar{D}$ and $\chi(x)=0$ for $\left|x_{2}\right|>\rho$. Then $V_{k} g=\chi V_{k} g+(1-\chi) V_{k} g$. Note that $(1-\chi) V_{k} g=\int_{D}(1-\chi) G(\cdot-y) g(y) \mathrm{d} y$ is an integral operator with a smooth kernel, since the series in (77) converges absolutely and uniformly for $\left|x_{2}\right| \geq \rho>0$, as well as all its partial derivatives. In consequence, the integral operator $(1-\chi) V_{k}$ is bounded from $L^{2}(D)$ into $H_{\alpha}^{2}\left(\Omega_{R}\right)$, since

$$
\left\|\partial_{1}^{\beta_{1}} \partial_{2}^{\beta_{2}}\left((1-\chi) V_{k} g\right)\right\|_{L^{2}\left(\Omega_{R}\right)}^{2} \leq \int_{\Omega_{R}} \int_{D}\left|\partial_{1}^{\beta_{1}} \partial_{2}^{\beta_{2}}\left[(1-\chi(x)) G_{k, \alpha}(x-y)\right]\right|^{2} \mathrm{~d} y \mathrm{~d} x\|g\|_{L^{2}(D)}^{2}
$$

for all $\beta_{1,2} \in \mathbb{N}$ such that $\beta_{1}+\beta_{2} \leq 2$. It remains to show the boundedness of $\chi V_{k}$ from $L^{2}(D)$ into $H^{2}\left(\Omega_{\rho}\right)$. Let $g \in L^{2}(D)$ and consider the operator $K_{2 \rho}$ from Proposition 4, mapping $L^{2}\left(\Omega_{2 \rho}\right)$ into $H_{\text {per }}^{2}\left(\Omega_{2 \rho}\right) \subset H_{\alpha}^{2}\left(\Omega_{2 \rho}\right)$,

$$
\left(K_{2 \rho} g\right)(x)=\int_{D} \mathcal{K}_{2 \rho}(x-y) g(y) \mathrm{d} y \quad \text { for } x \in \Omega_{2 \rho} .
$$

If $x \in \Omega_{\rho}$, then $\left|x_{2}-y_{2}\right| \leq 2 \rho$, that is, $\mathcal{K}_{2 \rho}(x-y)=G_{k, \alpha}(x-y)$. Hence, $K_{2 \rho} g=V_{k} g$ in $\Omega_{\rho}$, and hence $\chi K_{2 \rho} g=\chi V_{k} g$ in $\Omega_{\rho}$. Since $\chi$ is a smooth function, we conclude that $\chi V_{k}$ is bounded from $L^{2}(D)$ into $H_{\alpha}^{2}\left(\Omega_{\rho}\right)$. 
Note that the potential $V_{k} f$ can be extended to an $\alpha$-quasi-periodic function in $H_{\mathrm{loc}}^{2}\left(\mathbb{R}^{2}\right)$, due to the $\alpha$-quasi-periodicity of the kernel.

Lemma 6. For $g \in L^{2}\left(D, \mathbb{C}^{2}\right)$ the potential $w=\operatorname{div} V_{k} g$ belongs to $H_{\alpha}^{1}\left(\Omega_{\rho}\right)$ for all $\rho>0$. It is the unique radiating weak solution to $\Delta w+k^{2} w=-\operatorname{div} g$ in $\Omega$, that is, it satisfies

$$
\int_{\Omega}\left(\nabla w \cdot \nabla \bar{v}-k^{2} w \bar{v}\right) \mathrm{d} x=-\int_{D} g \cdot \nabla \bar{v} \mathrm{~d} x
$$

for all $v \in H_{\alpha}^{1}(\Omega)$ with compact support, and additionally the Rayleigh expansion condition (4).

Proof. Lemma 5 and $\alpha$-quasi-periodicity of the kernel of $V_{k}$ imply that $w$ is a function in $H_{\alpha}^{1}\left(\Omega_{\rho}\right)$ for all $\rho>0$. It is sufficient to prove (17) for all smooth $\alpha$-quasi-periodic test functions $v$ that are supported in $\left\{\left|x_{2}\right|<C\right\}$ for some $C>0$ depending on $v$. It is well-known that $p=V_{k} g \in H_{\alpha}^{2}(\Omega)$ is a weak solution to the Helmholtz equation, that is,

$$
\int_{\Omega}\left(\nabla p_{j} \cdot \nabla \partial_{j} \bar{v}-k^{2} p_{j} \partial_{j} \bar{v}\right) \mathrm{d} x=-\int_{D} g_{j} \partial_{j} \bar{v} \mathrm{~d} x
$$

for $j=1,2$. An integration by parts shows that

$$
\int_{\Omega}\left(\nabla \operatorname{div} p \cdot \nabla \bar{v}-k^{2} \operatorname{div} p \bar{v}\right) \mathrm{d} x=-\int_{D} g \cdot \nabla \bar{v} \mathrm{~d} x
$$

which implies (17) due to $\operatorname{div} p=w$. Since the components of the potential $p=V_{k} g$ satisfy the Rayleigh condition, a simple computation shows that the divergence $w=\operatorname{div} p$ does also satisfy the latter condition. Uniqueness of a radiating solution to (17) when $g=0$ follows from the Rayleigh expansion condition and a unique continuation argument.

Returning to the differential equation (3) for the scattered field $u^{s}$, let us set $f=Q \nabla u^{i}$. (Recall that $Q=\varepsilon_{\mathrm{r}}^{-1}-I_{2}$.) The variational formulation of (3) is

$$
\int_{\Omega}\left(\nabla u^{s} \cdot \nabla \bar{v}-k^{2} u^{s} \bar{v}\right) \mathrm{d} x=-\int_{D}\left(Q \nabla u^{s}+f\right) \cdot \nabla \bar{v} \mathrm{~d} x
$$

for all $v \in H_{\alpha}^{1}(\Omega)$ with compact support in $\bar{\Omega}$. From Lemma 6 we know that the radiating solution to this problem is given by $u^{s}=\operatorname{div} V_{k}\left(Q \nabla u^{s}+f\right)$. Hence, we aim to find $u^{s}$ : $\Omega \rightarrow \mathbb{C}$ that belongs to $H_{\alpha}^{1}\left(\Omega_{R}\right)$ for all $R>0$, such that

$$
u^{s}-\operatorname{div} V_{k}\left(Q \nabla u^{s}\right)=\operatorname{div} V_{k}(f) \quad \text { in } \Omega .
$$

\section{Gårding Inequalities in Weighted Sobolev Spaces}

For scattering problems in free space and for scalar and positive contrast, the paper [10] investigates integral equations similar to (19) in weighted spaces. In this section we generalize the results from [10] to anisotropic and possibly sign-changing coefficients in a periodic 
setting, proving a Gårding inequality for $I-\operatorname{div} V_{k}(Q \nabla \cdot)$ in a anisotropically weighted $\alpha$-quasi-periodic $H^{1}$-space.

From (19) it is obvious that the knowledge of $u$ in $D$ is sufficient to determine $u$ in $\Omega \backslash \bar{D}$ by integration. Thus, we define the operator $L_{k}: f \mapsto \operatorname{div} V_{k} f$ that is bounded from $L^{2}\left(D, \mathbb{C}^{2}\right)$ into $H_{\alpha}^{1}(D)$ and consider the integral equation

$$
u=L_{k}(Q \nabla u+f) \quad \text { in } H_{\alpha}^{1}(D) .
$$

To study Gårding inequalities for volume integral equations, we introduce suitable weighted Sobolev spaces. To this end, we recall that the symmetric $2 \times 2$ matrix $\operatorname{Re}(Q)$ has pointwise almost everywhere in $D$ an eigenvalue decomposition $\operatorname{Re}(Q)=U^{*} \Sigma U$ with a diagonal matrix $\Sigma$ and an orthogonal matrix $U$. This decomposition can be used to define the absolute value $|\operatorname{Re}(Q)|=U^{*}|\Sigma| U$ and the square root $|\operatorname{Re}(Q)|^{1 / 2}=U|\Sigma|^{1 / 2} U^{*}$, where the absolute value and the square root are element-wise applied to the diagonal matrix $\Sigma$. The two eigenvalues $\lambda_{1,2}$ of $\operatorname{Re}(Q)$ define

$$
\lambda_{\min }(x)=\min \left\{\left|\lambda_{1}(x)\right|,\left|\lambda_{2}(x)\right|\right\}, \quad \lambda_{\max }(x)=\max \left\{\left|\lambda_{1}(x)\right|,\left|\lambda_{2}(x)\right|\right\}, \quad x \in D .
$$

We assume in the following that $\operatorname{Re}(Q)$ is pointwise either strictly positive or strictly negative definite, such that we can assign a sign function $\operatorname{sign}(\operatorname{Re}(Q)) \in L^{\infty}(\Omega)$ to $\operatorname{Re}(Q)$, indicating whether the eigenvalues of $\operatorname{Re}(Q)$ are positive or negative. In the sequel, we write $\operatorname{Re}(Q) \geq c$ in $D(\operatorname{Re}(Q) \leq c$ in $D)$ to indicate that the eigenvalues $\lambda_{1,2}$ are larger than or equal to (less than or equal to) a constant $c$, almost everywhere in $D$. Note that the spectral matrix norm is denoted by $|\cdot|_{2}$.

We denote by $H_{\alpha, Q}^{1}(D)$ the completion of $H_{\alpha}^{1}(D)$ with respect to the norm $\|\cdot\|_{H_{\alpha, Q}^{1}(D)}$,

$$
\|u\|_{H_{\alpha, Q}^{1}(D)}^{2}:=\|\sqrt{|\operatorname{Re}(Q)|} \nabla u\|_{L^{2}\left(D, \mathbb{C}^{2}\right)}^{2}+\|u\|_{L^{2}(D)}^{2} .
$$

Since we assumed that $\operatorname{supp}(\operatorname{Re}(Q))=\bar{D}$, this norm is non-degenerate. Moreover, $\|u\|_{H_{\alpha, Q}^{1}(D)}$ is an equivalent norm in $H_{\alpha}^{1}(D)$ provided that $|\operatorname{Re}(Q)|$ is bounded from below in $D$ by some positive constant. In general, $\|u\|_{H_{\alpha, Q}^{1}(D)} \leq\left(1+\|\mid \sqrt{\mid \operatorname{Re}(Q)}\|_{2} \|_{L^{\infty}(D)}\right)\|u\|_{H_{\alpha}^{1}(D)}$. Note also that the norm of $H_{\alpha, Q}^{1}(D)$ is linked to the sesquilinear form

$$
a_{Q}(u, v)=\int_{D}[\operatorname{sign}(\operatorname{Re}(Q)) Q \nabla u \cdot \nabla \bar{v}+u \bar{v}] \mathrm{d} x, \quad u, v \in H_{\alpha, Q}^{1}(D) .
$$

Indeed, $\|u\|_{H_{\alpha, Q}^{1}(D)}^{2}=\operatorname{Re}\left[a_{Q}(u, u)\right]$ for $u \in H_{\alpha, Q}^{1}(D)$. In consequence, the form $a_{Q}$ is non-degenerate, that is, if $a_{Q}(u, v)=0$ for all $v \in H_{\alpha, Q}^{1}(D)$, then $u=0$.

If $\operatorname{Im} Q$ vanishes in $D$ (that is, the values of $x \mapsto Q(x)$ are self-adjoint matrices), then $a_{Q}$ is simply the inner product associated with the norm of $H_{\alpha, Q}^{1}(D)$,

$$
\langle u, v\rangle_{H_{\alpha, Q}^{1}(D)}=\int_{D}[|Q| \nabla u \cdot \nabla \bar{v}+u \bar{v}] \mathrm{d} x, \quad u, v \in H_{\alpha, Q}^{1}(D) .
$$


Lemma 7. Assume that there exists $C>0$ such that

$$
|\operatorname{Im}(Q(x)) \xi| \leq C|\operatorname{Re}(Q(x)) \xi| \quad \text { for almost every } x \in D \text { and all } \xi \in \mathbb{C}^{2} .
$$

Then $v \mapsto L_{k}(Q \nabla v)$ is bounded on $H_{\alpha, Q}^{1}(D)$.

Proof. Due to Theorem $5, L_{k}$ is bounded from $L^{2}\left(D, \mathbb{C}^{2}\right)$ into $H_{\alpha}^{1}(D)$. Furthermore, $v \mapsto$ $Q \nabla v$ is bounded from $H_{\alpha, Q}^{1}(D)$ into $L^{2}\left(D, \mathbb{C}^{2}\right)$, since

$$
\begin{aligned}
\|Q \nabla u\|_{L^{2}\left(D, \mathbb{C}^{2}\right)} & \leq\|\operatorname{Re}(Q) \nabla u\|_{L^{2}\left(D, \mathbb{C}^{2}\right)}+\|\operatorname{Im}(Q) \nabla u\|_{L^{2}\left(D, \mathbb{C}^{2}\right)} \\
& \leq\|\operatorname{Re}(Q) \mid \nabla u\|_{L^{2}\left(D, \mathbb{C}^{2}\right)}+C\|\operatorname{Re}(Q) \nabla u\|_{L^{2}\left(D, \mathbb{C}^{2}\right)} \\
& \leq(1+C)\|\mid \sqrt{\mid \operatorname{Re}(Q) \|}\|_{2}\left\|_{L^{\infty}(D)}\right\| u \|_{H_{\alpha, Q}^{1}(D)} .
\end{aligned}
$$

Moreover, the imbedding $H_{\alpha}^{1}(D) \subset H_{\alpha, Q}^{1}(D)$ is bounded, as mentioned above. Hence, $v \mapsto L_{k}(Q \nabla v)$ is bounded on $H_{\alpha, Q}^{1}(D)$.

Remark 8. Condition (24) is satisfied if the absolute values of the eigenvalues of $\operatorname{Im} Q$ are pointwise bounded by $C \lambda_{\min }$ (recall from (21) that $\lambda_{\min }$ is the minimum of the absolute values of the eigenvalues of $\operatorname{Re}(Q))$.

If $u \in H_{\alpha}^{1}(D) \subset H_{\alpha, Q}^{1}(D)$ solves the Lippmann-Schwinger equation (20), then Lemma (7) implies that $u$ solves the same equation in $H_{\alpha, Q}^{1}(D)$. Since $a_{Q}$ is non-degenerate, solving the Lippmann-Schwinger equation in $H_{\alpha, Q}^{1}(D)$ is equivalent to solve

$$
a_{Q}\left(u-L_{k}(Q \nabla u+f), v\right)=0 \quad \text { for all } v \in H_{\alpha, Q}^{1}(D) .
$$

If $u \in H_{\alpha, Q}^{1}(D)$ solves the latter variational problem for some $f \in L^{2}\left(D, \mathbb{C}^{2}\right)$, then $u=$ $L_{k}(Q \nabla u+f)$ belongs to $H_{\alpha}^{1}(D)$, due to (25) and since $L_{k}$ is bounded from $L^{2}\left(D, \mathbb{C}^{2}\right)$ into $H_{\alpha}^{1}(D)$.

Proposition 9. Assume that $f \in L^{2}\left(D, \mathbb{C}^{2}\right)$. Then any solution to the LippmannSchwinger equation (20) in $H_{\alpha}^{1}(D)$ is a solution in $H_{\alpha, Q}^{1}(D)$ and vice versa.

Our aim is now to prove a (generalized) Gårding inequality for the variational problem (26). The following lemma will turn out to be useful.

Lemma 10. Suppose that $X$ and $Y$ are Hilbert spaces. Let $T_{1,2}$ be bounded linear operators from $X$ into $Y$ and consider the sesquilinear form $a: X \times X \rightarrow \mathbb{C}$, defined by $a(u, v)=$ $\left\langle T_{1} u, T_{2} v\right\rangle_{Y}$ for $u, v \in X$. If one of the operators $T_{1}$ and $T_{2}$ is compact, then the linear operator $A: X \rightarrow X$, defined by $\langle A u, v\rangle_{X}=a(u, v)$ for all $u, v \in X$, is compact, too.

Proof. It is easily seen that $A$ is a well-defined bounded linear operator. Obviously, $\left|\langle A u, v\rangle_{X}\right|=|a(u, v)| \leq C\left\|T_{1} u\right\|_{Y}\left\|T_{2} v\right\|_{Y}$ for $u, v \in X$. Assume that $T_{1}$ is compact, and note that

$$
\|A u\|_{X}=\sup _{0 \neq v \in X} \frac{\left|\langle A u, v\rangle_{X}\right|}{\|v\|_{X}} \leq C\left\|T_{1} u\right\|_{Y}
$$


If a sequence $\left\{u_{n}\right\}$ converges weakly to zero in $X$, then $\left\{T_{1} u_{n}\right\}$ contains a strongly convergent subsequence tending to zero in $Y$. Consequently, $\left\{A u_{n}\right\}$ also contains a strongly convergent zero sequence, which means that $A$ is compact. One can analogously derive the compactness of $T$ in case that $T_{2}$ is compact, since $a(u, v)=\left\langle T_{2}^{*} T_{1} u, v\right\rangle$.

The next lemma proves Gårding inequalities for the operator $v \mapsto v-L_{k}(Q \nabla v)$ using the sesquilinear form $a_{Q}$ from (23). The second part of the claim uses a periodic extension operator

$$
E: H_{\alpha}^{1}(D) \rightarrow H_{\alpha}^{1}(\Omega),\left.\quad E(u)\right|_{D}=u,\left.\quad E(u)\right|_{\Omega \backslash \Omega_{2 \rho}}=0,
$$

introduced in Appendix B. The operator norm of $E$ is

$$
\|E\|_{H_{\alpha}^{1}(D) \rightarrow H_{\alpha}^{1}\left(\Omega_{2 \rho}\right)}=\left(1+\|E\|_{H_{\alpha}^{1}(D) \rightarrow H_{\alpha}^{1}\left(\Omega_{2 \rho} \backslash \bar{D}\right)}^{2}\right)^{1 / 2} .
$$

Theorem 11. Assume that $D$ is a Lipschitz domain and that $Q \in L^{\infty}\left(D, \mathbb{C}^{2 \times 2}\right)$.

(a) If $\operatorname{Re}(Q)>0$ in $D$, then there exists a compact operator $K_{+}$on $H_{\alpha, Q}^{1}(D)$ such that

$\operatorname{Re}\left[a_{Q}\left(v-L_{k}(Q \nabla v), v\right)\right] \geq\|v\|_{H_{\alpha, Q}^{1}(D)}^{2}-\operatorname{Re}\left\langle K_{+} v, v\right\rangle_{H_{\alpha, Q}^{1}(D)}, \quad v \in H_{\alpha, Q}^{1}(D)$.

(b) If $\operatorname{Re}(Q)<-1$, and if

$$
\|E\|_{H_{\alpha}^{1}(D) \rightarrow H_{\alpha}^{1}\left(\Omega_{2 \rho}\right)}<\inf _{D}|\operatorname{Re}(Q)|_{2}^{1 / 2},
$$

then there exists a constant $C>0$ and a compact operator $K_{-}$on $H_{\alpha, Q}^{1}(D)$ such that

$$
-\operatorname{Re}\left[a_{Q}\left(v-L_{k}(Q \nabla v), v\right)\right] \geq C\|v\|_{H_{\alpha, Q}^{1}(D)}^{2}-\operatorname{Re}\left\langle K_{-} v, v\right\rangle_{H_{\alpha, Q}^{1}(D)}, \quad v \in H_{\alpha, Q}^{1}(D) .
$$

Remark 12. If $\operatorname{Im}(Q)=0$ in $D$, then both statements (27) and (29) are nothing but standard Gärding estimates: The form $a_{Q}$ defines an inner product on $H_{\alpha, Q}^{1}(D)$, and, e.g., (27) can be rewritten as $\operatorname{Re}\left\langle v-L_{k}(Q \nabla v), v\right\rangle \geq\|v\|^{2}-\operatorname{Re}\left\langle K_{+} v, v\right\rangle$ for $v \in H_{\alpha, Q}^{1}(D)$. Proof. (a) We start with the case $\operatorname{Re}(Q)>0$ in $D$. Let $v \in H_{\alpha, Q}^{1}(D)$ and define $w$ by

$$
w=L_{\mathrm{i}}(Q \nabla v)=\operatorname{div} \int_{D} G_{\mathrm{i}, \alpha}(\cdot-y)[Q(y) \nabla v(y)] \mathrm{d} y \quad \text { in } \Omega .
$$

Then $w \in H_{\alpha}^{1}(\Omega)$ decays exponentially to zero as $\left|x_{2}\right|$ tends to infinity. Moreover, $\Delta w-w=$ $-\operatorname{div}(Q \nabla v)$ holds in $\Omega$ in the weak sense due to Lemma 6, that is,

$$
\int_{\Omega}[\nabla w \cdot \nabla \bar{\psi}+w \bar{\psi}] \mathrm{d} x=-\int_{D} Q \nabla v \cdot \nabla \bar{\psi} \mathrm{d} x \quad \text { for all } \psi \in H_{\alpha}^{1}(\Omega) .
$$

Setting $\psi=w$, we find that $-\operatorname{Re} \int_{D} Q \nabla v \cdot \nabla \bar{w} \mathrm{~d} x=\|w\|_{H^{1}(\Omega)}^{2}$. Hence,

$$
\begin{aligned}
\operatorname{Re}\left[a_{Q}\left(v-L_{\mathrm{i}}(Q \nabla v), v\right)\right] & =\int_{D}\left[\operatorname{Re}(Q) \nabla v \cdot \nabla \bar{v}+|v|^{2}\right] \mathrm{d} x-\operatorname{Re} \int_{D}[Q \nabla w \cdot \nabla \bar{v}+w \bar{v}] \mathrm{d} x \\
& =\int_{D}\left[|\sqrt{\operatorname{Re}(Q)} \nabla v|^{2}+|v|^{2}-\operatorname{Re}(w \bar{v})\right] \mathrm{d} x+\int_{\Omega}\left[|\nabla w|^{2}+|w|^{2}\right] \mathrm{d} x \\
& \geq\|v\|_{H_{\alpha, Q}^{1}(D)}^{2}-\frac{1}{2}\|v\|_{L^{2}(D)}^{2}+\frac{1}{2} \int_{D}\left[|v|^{2}+|w|^{2}-2 \operatorname{Re}(w \bar{v})\right] \mathrm{d} x,
\end{aligned}
$$


where the last term on the right is positive. In consequence,

$$
\operatorname{Re}\left[a_{Q}\left(v-L_{k}(Q \nabla v), v\right)\right] \geq\|v\|_{H_{\alpha, Q}^{1}(D)}^{2}-\frac{1}{2}\langle v, v\rangle_{L^{2}(D)}-\operatorname{Re}\left[a_{Q}\left(\left(L_{k}-L_{\mathrm{i}}\right)(Q \nabla v), v\right)\right]
$$

for all $v \in H_{\alpha, Q}^{1}(D)$. Due to Lemma 10 and Rellich's lemma there exists a compact operator $K_{1}$ on $H_{\alpha, Q}^{1}(D)$ such that $\langle v, v\rangle_{L^{2}(D)}=2 \operatorname{Re}\left\langle K_{1} v, v\right\rangle_{H_{\alpha, Q}^{1}(D)}$. Further, the operator $\left(L_{k}-\right.$ $\left.L_{\mathrm{i}}\right)(Q \nabla \cdot)$ is compact on $H_{\alpha}^{1}(D)$ due to the smoothness of the kernel shown in Appendix $\mathrm{A}$, Hence the operator $K_{2}$ defined by $\left\langle K_{2} v, v\right\rangle_{H_{\alpha, Q}^{1}(D)}=a_{Q}\left(\left(L_{k}-L_{\mathrm{i}}\right)(Q \nabla v), v\right)$ is compact on $H_{\alpha, Q}^{1}(D)$ due to Lemma 10 and the boundedness of the imbedding $H_{\alpha}^{1}(D) \subset H_{\alpha, Q}^{1}(D)$. Setting $K_{+}:=K_{1}+K_{2}$, we obtain the claimed generalized Gårding inequality.

(b) Now we consider the case that $\operatorname{Re}(Q)<-1$ in $D$, and assume additionally that (28) holds. As in the first part of the proof, the variational formulation (31) for $w$, defined as in (30), yields that

$$
\begin{aligned}
-\operatorname{Re}\left[a_{Q}\left(v-L_{\mathrm{i}}(Q \nabla v), v\right)\right] & =\operatorname{Re} \int_{D}\left[\operatorname{Re}(Q) \nabla v \cdot \nabla \bar{v}-|v|^{2}-Q \nabla w \cdot \nabla \bar{v}+w \bar{v}\right] \mathrm{d} x \\
& =-\int_{D}\left[|\sqrt{|\operatorname{Re}(Q)|} \nabla v|^{2}+|v|^{2}\right] \mathrm{d} x+\|w\|_{H_{\alpha}^{1}(\Omega)}^{2}+\operatorname{Re} \int_{D} w \bar{v} \mathrm{~d} x \\
& \geq\|w\|_{H_{\alpha}^{1}(\Omega)}^{2}-\|v\|_{H_{\alpha, Q}^{1}(D)}^{2}+\operatorname{Re} \int_{D} w \bar{v} \mathrm{~d} x .
\end{aligned}
$$

We plug in $\psi=-E(v)$ into (31) and take the real part of that equation, to find that

$$
\begin{aligned}
\|\sqrt{|\operatorname{Re}(Q)|} \nabla v\|_{L^{2}\left(D, \mathbb{C}^{2}\right)}^{2} & \leq\|w\|_{H_{\alpha}^{1}(\Omega)}\|E(v)\|_{H_{\alpha}^{1}(\Omega)} \leq\|E\|_{H_{\alpha}^{1}(D) \rightarrow H_{\alpha}^{1}\left(\Omega_{2 \rho}\right)}\|w\|_{H_{\alpha}^{1}(\Omega)}\|v\|_{H_{\alpha}^{1}(D)} \\
& \leq\|E\|\|w\|_{H_{\alpha}^{1}(\Omega)}\left(\left\|\left|\sqrt{|\operatorname{Re}(Q)|}^{-1}\right|_{2}\right\|_{L^{\infty}(D)}\|v\|_{H_{\alpha, Q}^{1}(D)}+\|v\|_{L^{2}(D)}\right) .
\end{aligned}
$$

For $x \in D$, the spectral matrix norm $\left|\sqrt{|\operatorname{Re}(Q)|}^{-1}(x)\right|_{2}$ of the inverse of $\sqrt{|\operatorname{Re}(Q)|}(x)$ equals the reciprocal value $\lambda_{\min }(x)^{-1 / 2}\left(\lambda_{\min , \max }\right.$ are the smallest/largest eigenvalue, in magnitude, of $\operatorname{Re}(Q)$, see (21)). Note that

$$
\begin{aligned}
\left\|\left|\sqrt{|\operatorname{Re}(Q)|}^{-1}\right|_{2}\right\|_{L^{\infty}(D)}^{-1} & =\left[\sup _{x \in D} \lambda_{\min }(x)^{-1 / 2}\right]^{-1}=\inf _{x \in D} \lambda_{\min }(x)^{1 / 2} \leq \sup _{x \in D} \lambda_{\max }(x)^{1 / 2} \\
& \leq\left[1+\sup _{x \in D} \lambda_{\max }(x)\right]^{1 / 2}=\left[1+\left\||\operatorname{Re}(Q)|_{2}\right\|_{L^{\infty}(D)}\right]^{1 / 2} .
\end{aligned}
$$

Next, we estimate that

$$
\begin{gathered}
\|v\|_{H_{\alpha, Q}^{1}(D)}^{2}-\left[1+\left\|\left.\operatorname{Re}(Q)\right|_{2}\right\|_{L^{\infty}(D)}\right]\|v\|_{L^{2}(D)}^{2} \leq\|v\|_{H_{\alpha, Q}^{1}(D)}^{2}-\|v\|_{L^{2}(D)}^{2} \\
\leq\|E\|\left\|\left|\sqrt{|\operatorname{Re}(Q)|}^{-1}\right|_{2}\right\|_{L^{\infty}(D)}\|w\|_{H_{\alpha}^{1}(\Omega)} \\
\left(\|v\|_{H_{\alpha, Q}^{1}(D)}+\left[1+\left\||\operatorname{Re}(Q)|_{2}\right\|_{L^{\infty}(D)}\right]^{1 / 2}\|v\|_{L^{2}(D)}\right) .
\end{gathered}
$$

Dividing by the term in brackets on the right, we obtain that

$$
\|v\|_{H_{\alpha, Q}^{1}(D)}-\left[1+\left\||\operatorname{Re}(Q)|_{2}\right\|_{\infty}\right]^{1 / 2}\|v\|_{L^{2}(D)} \leq\|E\|\left\|\left|\sqrt{|\operatorname{Re}(Q)|}^{-1}\right|_{2}\right\|_{L^{\infty}(D)}\|w\|_{H_{\alpha}^{1}(\Omega)} .
$$


Note that the constant

$$
c:=\|E\|_{H_{\alpha}^{1}(D) \rightarrow H_{\alpha}^{1}\left(\Omega_{2 \rho}\right)}\left\|\left|\sqrt{|\operatorname{Re}(Q)|}^{-1}\right|_{2}\right\|_{L^{\infty}(D)}
$$

is by assumption (28) less than one. If we set for a moment, $C=\left[1+\left\||\operatorname{Re}(Q)|_{2}\right\|_{\infty}\right]^{1 / 2}$ then (32) and Cauchy's inequality imply that

$$
\begin{aligned}
c^{2}\|w\|_{H_{\alpha}^{1}(\Omega)}^{2} & \geq\|v\|_{H_{\alpha, Q}^{1}(D)}^{2}+C^{2}\|v\|_{L^{2}(D)}^{2}-2 C\|v\|_{H_{\alpha, Q}^{1}(D)}\|v\|_{L^{2}(D)} \\
& \geq\left(1-\varepsilon^{2}\right)\|v\|_{H_{\alpha, Q}^{1}(D)}^{2}+C^{2}\left(1-1 / \varepsilon^{2}\right)\|v\|_{L^{2}(D)}^{2}, \quad \varepsilon \in(0,1) .
\end{aligned}
$$

In consequence,

$$
\begin{aligned}
-\operatorname{Re}\left[a_{Q}\left(v-L_{k}(Q \nabla v), v\right)\right] \geq\left(\frac{1-\varepsilon^{2}}{c^{2}}-1\right)\|v\|_{H_{\alpha, Q}^{1}(D)}^{2} \\
-\operatorname{Re} \int_{D} w \bar{v} \mathrm{~d} x+C^{2} \frac{\varepsilon^{2}-1}{(c \varepsilon)^{2}}\|v\|_{L^{2}(D)}^{2}+\operatorname{Re}\left[a_{Q}\left(\left(L_{k}-L_{\mathrm{i}}\right)(Q \nabla v), v\right)\right]
\end{aligned}
$$

for $\varepsilon \in(0,1)$. Since $c<1$ there exists $\varepsilon \in(0,1)$ such that $1-\varepsilon^{2}>c^{2}$, that is, $\left(1-\varepsilon^{2}\right) / c^{2}-1>$ 0 . The last three terms on the right-hand side of (33) can then be treated as compact perturbations, in a similar way as in the proof of the first part.

Remark 13. (a) If $\operatorname{Re}(Q)<-1$ in $D$, then solutions to $\operatorname{div}\left(\left(I_{2}+Q\right) \nabla u\right)+k^{2} u$ decay exponentially in D. If not only the electric permittivity but also the magnetic permeability changed sign, then the corresponding solution would not decay, yielding a possibly more interesting metamaterial. Volume integral equations for such structures yield operator equations combining $L_{k}$ and $V_{k}$, see, e.g., [10]. Since $V_{k}$ is compact on $H^{1}$, the above Garding inequalities extend to this setting. For simplicity, we restrict ourselves here to the nonmagnetic case.

(b) In the last result, we assumed that the sign of $\operatorname{Re}(Q)$ is constant in $D$. It is possible to treat sign changes of the contrast function in $D$, but the simple choice $\psi=-E(v)$ that we plugged in the second part of the proof into (31) has to be adapted.

It is a standard result that the Gårding inequalities from the last theorem imply the following consequences for the solvability of the integral equation and the scattering problem.

Theorem 14. Suppose that the assumptions of Theorem 11(a) or (b) hold, that the boundedness condition (24) holds, and that the homogeneous equation $v-L_{k}(Q \nabla v)=0$ in $H_{\alpha, Q}^{1}(D)$ has only the trivial solution. Then (20) has a unique solution for all $f \in L^{2}\left(D, \mathbb{C}^{2}\right)$. If $f=Q \nabla u^{i}$, then this solution can be extended by the right-hand side of (20) to a solution to the variational formulation of the scattering problem (5). Especially, if the integral equation is uniquely solvable in $H_{\alpha, Q}^{1}(D)$, then (5) is uniquely solvable in $H_{\alpha}^{1}\left(\Omega_{\rho}\right)$. 


\section{Gårding Inequalities in Standard Sobolev Spaces}

The generalized Gårding inequalities from the last section imply Grarding inequalities in the standard unweighted periodic Sobolev space $H_{\alpha}^{1}(D)$ if the material parameter $\varepsilon_{\mathrm{r}}$ (or, equivalently, the contrast), is isotropic. Hence, in this section we assume that the contrast is a scalar real-valued function $q$, that is,

$$
Q=q I_{2} \quad \text { in } \Omega \text {. }
$$

As above, $\bar{D}$ is the support of $q$. Under this assumption we denote the weighted Sobolev spaces from (22) by $H_{\alpha, q}^{1}(D)$, and their norm by

$$
\|u\|_{H_{\alpha, q}^{1}(D)}:=\left(\|\sqrt{|\operatorname{Re}(q)|} \nabla u\|_{L^{2}\left(D, \mathbb{C}^{2}\right)}^{2}+\|u\|_{L^{2}(D)}^{2}\right)^{1 / 2} .
$$

Since $q$ is real-valued, the form $a_{q}$ from $(23)$ is the inner product of $H_{\alpha, q}^{1}(D)$, and the generalized Gårding inequalities from the last section directly transform to standard ones. Again, we assume that the sign of $q$ is constant in $D$. Since we use regularity theory to prove compactness of certain commutators, we will need to require more smoothness of $q$ and $D$ compared to the results in the last section.

Lemma 15. Assume that $D$ is a domain of class $C^{2,1}$ and that $\mu \in C^{2,1}(\bar{D}), 2 \pi$-periodic in $x_{1}$. Then $T: H_{\alpha}^{1}(D) \rightarrow H_{\alpha}^{1}(D)$ defined by $T v:=\operatorname{div}\left[\mu V_{k}(q \nabla(v / \mu))-V_{k}(q \nabla v)\right]$ is a compact operator.

Proof. We denote by $\mu^{*} \in C^{2,1}\left(\overline{\Omega_{\rho}}\right)$ a periodic extension of $\mu \in C^{2,1}(\bar{D})$ to $\Omega_{\rho}$ (see Appendix $\mathrm{B}$ on periodic extension operators). Then $\left.\mu^{*}\right|_{D}=\mu$. Consider the two $\alpha$-quasiperiodic functions

$$
w_{1}=V_{k}(q \nabla(v / \mu)) \quad \text { and } \quad w_{2}=V_{k}(q \nabla v) \quad \text { in } \Omega_{\rho} .
$$

Both functions satisfy differential equations,

$$
\Delta\left(\mu^{*} w_{1}\right)+k^{2}\left(\mu^{*} w_{1}\right)= \begin{cases}-q \mu \nabla(v / \mu)+2 \nabla \mu \cdot \nabla w_{1}+w_{1} \Delta \mu & \text { in } D \\ 2 \nabla \mu^{*} \cdot \nabla w_{1}+w_{1} \Delta \mu^{*} & \text { in } \Omega_{\rho} \backslash \bar{D},\end{cases}
$$

and $\Delta w_{2}+k^{2} w_{2}=-q \nabla v$ in $D$ and $\Delta w_{2}+k^{2} w_{2}=0$ in $\Omega_{\rho} \backslash \bar{D}$. Hence, $w=\mu^{*} w_{1}-w_{2}$ solves

$$
\Delta w+k^{2} w= \begin{cases}-q \mu \nabla(1 / \mu) v+2 \nabla \mu \cdot \nabla w_{1}+w_{1} \Delta \mu=: g_{1} & \text { in } D, \\ w_{1} \Delta \mu^{*}+2 \nabla \mu^{*} \cdot \nabla w_{1}=: g_{2} & \text { in } \Omega_{\rho} \backslash \bar{D} .\end{cases}
$$

The functions $g_{1}$ and $g_{2}$ belong to $H_{\alpha}^{1}(D)$ and $H_{\alpha}^{1}\left(\Omega_{\rho} \backslash \bar{D}\right)$, respectively. Their norms in these spaces are bounded by the norm of $\mu$ in $C^{2,1}(\bar{D})$ times the norm of $v$ in $H_{\alpha}^{1}(D)$. Due to Lemma 5 , the jump of the trace and the normal trace of $w_{1,2}$ across $\partial D$ vanishes. Hence, the Cauchy data of $w$ are also continuous across the boundary of $D$.

Since the volume potential $V_{k}$ is bounded from $L^{2}(D)$ into $H_{\alpha}^{2}(D)$, it is clear that $w$ belongs to $H_{\alpha}^{2}(D)$. The smoothness assumptions on $D$ and $\mu$ moreover allow to apply elliptic 
transmission regularity results [11, Theorem 4.20] to conclude that $w$ is even smoother than $H^{2}$. These regularity results will in turn imply the compactness of the operator $T: v \mapsto \operatorname{div} w$ on $H_{\alpha}^{1}(D)$. A straightforward adaption of the transmission regularity result [11, Theorem 4.20] to the periodic setting shows that

$$
\|w\|_{H^{3}(D)} \leq C\left[\|w\|_{H^{1}\left(\Omega_{\rho}\right)}+\left\|g_{1}\right\|_{H^{1}(D)}+\left\|g_{2}\right\|_{H^{1}\left(\Omega_{\rho} \backslash \bar{D}\right)}\right] \leq C\|v\|_{H_{\alpha}^{1}(D)} .
$$

The following lemma shows that the Gårding inequalities in the weighted spaces $H_{\alpha, q}^{1}(D)$ can be transformed into estimates in $H_{\alpha}^{1}(D)$ if, roughly speaking, the real-valued contrast $q$ is smooth enough and if $(\nabla q) / q$ is bounded.

Theorem 16. Assume that the scalar contrast $q$ is real-valued, that $|q| \geq q_{0}>0$ in $D$, and that $\sqrt{|q|} \in C^{2,1}(\bar{D})$. Moreover, assume that $D$ is of class $C^{2,1}$.

(a) If $q>0$ there exists a compact operator $K_{+}$on $H_{\alpha}^{1}(D)$ such that

$$
\operatorname{Re}\left\langle v-L_{k}(q \nabla v), v\right\rangle_{H_{\alpha}^{1}(D)} \geq\|v\|_{H_{\alpha}^{1}(D)}^{2}-\operatorname{Re}\left\langle K_{+} v, v\right\rangle_{H_{\alpha}^{1}(D)}, \quad v \in H_{\alpha}^{1}(D) .
$$

(b) If $q<0$, and if

$$
\|E\|_{H_{\alpha}^{1}(D) \rightarrow H_{\alpha}^{1}\left(\Omega_{2 \rho}\right)}<\inf _{D}|q|^{1 / 2}
$$

then there exists a compact operator $K_{-}$on $H_{\alpha}^{1}(D)$ such that

$$
-\operatorname{Re}\left\langle v-L_{k}(q \nabla v), v\right\rangle_{H_{\alpha}^{1}(D)} \geq C\|v\|_{H_{\alpha}^{1}(D)}^{2}-\operatorname{Re}\left\langle K_{-} v, v\right\rangle_{H_{\alpha}^{1}(D)}, \quad v \in H_{\alpha}^{1}(D),
$$

where $C$ is the constant from (29).

Proof. We only prove case (a) here, supposing that $q>q_{0}>0$ in $D$. The proof for case (b) is analogous, essentially one needs to replace $\sqrt{q}$ by $\sqrt{|q|}$. For simplicity, let us from now on abbreviate

$$
\mu:=\sqrt{q} \in C^{2,1}(\bar{D}) .
$$

Choose an arbitrary $u \in H_{\alpha}^{1}(D)$ and consider $v=u / \mu$. Our assumptions on $q$ imply that $v \in H_{\alpha, q}^{1}(D)$, since $\|v\|_{H_{\alpha, q}^{1}(D)}^{2} \leq\left(2+\|1 / \mu\|_{\infty}^{2}+2\|(\nabla \mu) / \mu\|_{\infty}^{2}\right)\|u\|_{H_{\alpha}^{1}(D)}^{2}$. In Theorem 11(a) (see also Remark 12) we showed that $\operatorname{Re}\left\langle v-L_{k}(q \nabla v), v\right\rangle_{H_{\alpha, q}^{1}(D)} \geq\|v\|_{H_{\alpha, q}^{1}(D)}^{2}-$ $\operatorname{Re}\left\langle K_{1} v, v\right\rangle_{H_{\alpha, q}^{1}(D)}$ for a compact operator $K_{1}$ on $H_{\alpha, q}^{1}(D)$. This implies that

$$
\begin{aligned}
\operatorname{Re}\left\langle u-L_{k}(q \nabla u), u\right\rangle_{H_{\alpha}^{1}(D)} \geq\|u\|_{H_{\alpha}^{1}(D)}^{2}+ & \operatorname{Re}\left\langle K_{1}(u / \mu), u / \mu\right\rangle_{H_{\alpha, q}^{1}(D)} \\
& +\operatorname{Re}\left\langle K_{2} u, \nabla u\right\rangle_{L^{2}\left(D, \mathbb{C}^{2}\right)}+\operatorname{Re}\left\langle K_{3} u, u\right\rangle_{L^{2}(D)},
\end{aligned}
$$

with operators

$$
K_{2} u=\nabla\left[\operatorname{div}\left[\mu V_{k}(q \nabla(u / \mu))-V_{k}(q \nabla u)\right]\right]-\nabla\left[\nabla \mu \cdot V_{k}(q \nabla(u / \mu))\right]+(\nabla \mu) L_{k}(q \nabla(u / \mu))
$$


and

$$
K_{3} u=q \nabla(1 / \mu) \cdot\left[\nabla L_{k}(q \nabla(u / \mu))\right]+L_{k}(q \nabla(u / \mu)) / \mu-L_{k}(q \nabla u) .
$$

Lemma 15, the smoothness of $q$, and the boundedness of $V_{k}$ and $L_{k}$ from $L^{2}(D)$ and $L^{2}\left(D, \mathbb{C}^{2}\right)$ into $H_{\alpha}^{2}(D)$ and $H_{\alpha}^{1}(D)$, respectively, show that $K_{2}$ and $K_{3}$ are compact and bounded from $H_{\alpha}^{1}(D)$ into $L^{2}(D)$, respectively. Then the compact embedding $H_{\alpha}^{1}(D) \subset$ $L^{2}(D)$ and an application of Lemma 10 imply the claim.

Remark 17. The regularity assumptions on $\partial D$ and $q$ can be weakened using more sophisticated regularity results. It is for instance possible to treat piecewise smooth $q$ by an analogous technique. We do not discuss this issue to avoid technicalities that would not add new ideas.

Theorem 18. Suppose that $q$ and $D$ satisfy the assumptions of Theorem 16(a) or (b), and that the homogeneous equation $v-L_{k}(q \nabla v)=0$ in $H_{\alpha, q}^{1}(D)$ has only the trivial solution. Then (20) has a unique solution for all $f \in L^{2}\left(D, \mathbb{C}^{2}\right)$. If $f=q \nabla u^{i}$, then this solution can be extended by the right-hand side of (20) to a solution to the scattering problem (5). Especially, if the integral equation is uniquely solvable, then (20) is also uniquely solvable.

\section{A Smoothness of the Difference of Periodic Green's Func- tions}

The following lemma is a consequence of the corresponding result for the fundamental solution to the Helmholtz equation in free-space.

Lemma 19. Assume that $k^{2} \neq \alpha_{j}^{2}$ for all $j \in \mathbb{Z}$. Then the difference $G_{k, \alpha}-G_{\mathrm{i}, \alpha}$ can be written as

$$
G_{k, \alpha}(x)-G_{\mathrm{i}, \alpha}(x)=\alpha\left(|x|^{2}\right)+C|x|^{2} \ln (|x|) \beta\left(|x|^{2}\right)
$$

where $\alpha$ and $\beta$ are analytic functions and $C$ is a constant.

The smoothness of the difference $G_{k, \alpha}-G_{\mathrm{i}, \alpha}$ implies the following compactness statement for the corresponding volume potentials.

Corollary 20. Assume that $k^{2} \neq \alpha_{j}^{2}$ for all $j \in \mathbb{Z}$. Then $L_{k}-L_{\mathrm{i}}$ is compact on $H_{\alpha}^{1}(D)$.

\section{B Periodic Extension Operators}

In this section, we exemplary show how to construct a periodic extension operator

$$
E: H_{\alpha}^{1}(D) \rightarrow H_{\alpha}^{1}(\Omega),\left.\quad E(u)\right|_{D}=u,\left.\quad E(u)\right|_{\Omega \backslash \Omega_{2 \rho}}=0,
$$

that is used in Theorem [11. We will only construct $E$ for the case that the boundary of $D=\left\{\left(x_{1}, x_{2}\right)^{\top}: x_{1} \in(-\pi, \pi), \zeta_{-}\left(x_{1}\right)<x_{2}<\zeta_{+}\left(x_{1}\right)\right\}$ is given by two $2 \pi$-periodic Lipschitz continuous functions $\zeta_{ \pm}: \mathbb{R} \rightarrow(-\rho, \rho)$ such that $\zeta_{-}<-2 \rho / 3, \zeta_{+}>2 \rho / 3$, and 
$\left|\zeta_{ \pm}\left(x_{1}\right)-\zeta_{ \pm}\left(x_{1}^{\prime}\right)\right| \leq M\left|x_{1}-x_{1}^{\prime}\right|$ for $x_{1}, x_{1}^{\prime} \in \mathbb{R}$. The general case can be tackled using local patches as in [11, Appendix A].

For $u \in H_{\alpha}^{1}(D)$, we define

$$
v\left(x_{1}, x_{2}\right)= \begin{cases}u\left(x_{1}, 2 \zeta_{+}\left(x_{1}\right)-x_{2}\right) & \text { if } \zeta_{+}\left(x_{1}\right)<x_{2}<2 \zeta_{+}\left(x_{1}\right)-\zeta_{-}\left(x_{1}\right), \\ u\left(x_{1}, x_{2}\right) & \text { if } \zeta_{-}\left(x_{1}\right)<x_{2}<\zeta_{+}\left(x_{1}\right), \\ u\left(x_{1}, 2 \zeta_{-}\left(x_{1}\right)-x_{2}\right) & \text { if } 2 \zeta_{-}\left(x_{1}\right)-\zeta_{+}\left(x_{1}\right)<x_{2}<\zeta_{-}\left(x_{1}\right) .\end{cases}
$$

Note that $2 \zeta_{+}\left(x_{1}\right)-\zeta_{-}\left(x_{1}\right)>2 \rho$ and that $2 \zeta_{-}\left(x_{1}\right)-\zeta_{+}\left(x_{1}\right)<-2 \rho$. Straightforward computations show that $\|v\|_{H^{1}\left(\Omega_{2 \rho}\right)} \leq \max (\sqrt{3}, 2 \sqrt{2} M)\|u\|_{H_{\alpha}^{1}(D)}$, and the definition of $v$ implies that this function is $\alpha$-quasi-periodic.

To define the periodic extension operator, we use a smooth cut-off function $\chi: \mathbb{R} \rightarrow \mathbb{R}$, that satisfies $0 \leq \chi \leq 1, \chi\left(x_{2}\right)=1$ for $\left|x_{2}\right| \leq \rho$, and $\chi(2 \rho)=0$ for $\left|x_{2}\right| \geq 2 \rho$. Then we set

$$
E(u)=w, \quad w(x)= \begin{cases}\chi\left(x_{2}\right) v(x) & \text { for } x \in \Omega_{2 \rho} \\ 0 & \text { else }\end{cases}
$$

\section{References}

[1] A.-S. Bonnet-Ben Dhia, L. Chesnel, and P. Ciarlet Jr., Optimality of Tcoercivity for scalar interface problems between dielectrics and metamaterials, Preprint, (2011).

[2] A.-S. Bonnet-Ben Dhia, P. Ciarlet Jr., And C. M.Zwölf, Two-field and threefield formulations for wave transmission between media with opposite sign dielectric constants, J. Comput. Appl. Math., 204 (2007), pp. 408-417.

[3] — Time harmonic wave diffraction problems in materials with sign-shifting coefficients, J. Comput. Appl. Math., 234(6) (2010), pp. 1912-1919.

[4] A.-S. Bonnet-Ben Dhia And F. Starling, Guided waves by electromagnetic gratings and non-uniqueness examples for the diffraction problem, Math. Meth. Appl. Sci., 17 (1994), pp. 305-338.

[5] M. Costabel, E. Darrigrand, and E. Koné, Volume and surface integral equations for electromagnetic scattering by a dielectric body, J. Comput. Appl. Math, 234 (2010), pp. 1817-1825.

[6] M. Costabel, E. Darrigrand, And H. Sakly, The essential spectrum of the volume integral operator in electromagnetic scattering by a homogeneous body. Nov. 2011.

[7] J. Elschner And G. SChmidt, Diffraction of periodic structures and optimal design problems of binary gratings. Part I: Direct problems and gradient formulas, Math. Meth. Appl. Sci., 21 (1998), pp. 1297-1342. 
[8] T. Hohage, On the numerical solution of a three-dimensional inverse medium scattering problem, Inverse Problems, 17 (2001), pp. 1743-1763.

[9] A. Kirsch, Diffraction by periodic structures, in Proc. Lapland Conf. on Inverse Problems, L. Pävarinta and E. Somersalo, eds., Springer, 1993, pp. 87-102.

[10] A. Kirsch And A. Lechleiter, The operator equations of Lippmann-Schwinger type for acoustic and electromagnetic scattering problems in $L^{2}$, Applicable Analysis, 88 (2009), pp. 807-830.

[11] W. McLean, Strongly Elliptic Systems and Boundary Integral Operators, Cambridge University Press, Cambridge, UK, 2000.

[12] R. Pотthast, Electromagnetic scattering from an orthotropic medium, J. Int. Eq and Appl., 11 (1999), pp. 179-215.

[13] J. Richmond, Scattering by a dielectric cylinder of arbitrary cross section shape, IEEE Trans. Antennas Propag., 13 (1965), pp. 334-341.

[14] — TE-wave scattering by a dielectric cylinder of arbitrary cross-section shape, IEEE Trans. Antennas Propag., 14 (1966), pp. 460-464.

[15] K. SANDFORT, The factorization method for inverse scattering from periodic inhomogeneous media, PhD thesis, Karlsruher Institut für Technologie, 2010.

[16] J. Saranen And G. VAinikko, Periodic integral and pseudodifferential equations with numerical approximation, Springer, 2002.

[17] V. M. Shalaev, Optical negative-index metamaterials, Nature Photonics, 1 (2007), pp. $41-48$.

[18] G. VAinikKo, Fast solvers of the Lippmann-Schwinger equation, in Direct and Inverse Problems of Mathematical Physics, D. Newark, ed., Int. Soc. Anal. Appl. Comput. 5, Dordrecht, 2000, Kluwer, p. 423. 